\title{
Atomistic Study of Model CdTe Grain Boundaries
}

Tadas Paulauskas, ${ }^{1}$ Fatih Sen, ${ }^{2}$ Cyrus Sun, ${ }^{3}$ Edward Barnard, ${ }^{4}$ Moon Kim ${ }^{3}$, Sivananthan Sivalingham, ${ }^{1}$ Maria Chan, ${ }^{2}$ and Robert Klie ${ }^{1}$

1. University of Illinois at Chicago, Department of Physics, Chicago, IL

2. University of Texas at Dallas, Department of Materials Science and Engineering, Dallas, TX

3. Argonne National Laboratory, Center for Nanoscale Materials, Argonne, IL

4. Lawrence Berkeley National Laboratory, Molecular Foundry, Berkeley, CA

Poly-crystalline CdTe-based thin film photovoltaic (PV) devices are the forerunners in commercialized solar cell technology. Despite the commercial success, best laboratory cells achieve $21.5 \%$ [1]. Such cell performances are still far from the theoretical limits ( 30\%),[2] and improvements have mostly been due to better light collection, rather than improvements in the absorber layer and the open circuit voltage, $V_{\mathrm{oc}}$. Low $\mathrm{V}_{\mathrm{oc}}$ is widely believed to stem from poor back-contacts, and also from CdTe crystal defects, including point defects and numerous randomly oriented grain boundaries [3]. They tend to introduce localized nonradiative carrier recombination centers affecting the minority lifetimes ( 1-10ns in CdTe). Better atomicscale understanding of these defects is needed in order to further improve efficiency of the poly-CdTe solar cells.

In this collaborative research project, we investigate effects of the grain boundaries via ultra-high-vacuum (UHV) bonded CdTe bicrystals. Characterization is carried out in the aberration-corrected cold-field emission JEOL JEM-ARM200CF scanning transmission electron microscope (STEM) using high-angle annular dark field (HAADF) and annular bright field (ABF) imaging. Chemical composition of interfaces and dislocation cores is demonstrated via atomic-column-resolved X-ray energy dispersive spectroscopy (XEDS) using windowless silicon drift detector. Electronic defect structures were studied using firstprinciples density functional theory (DFT) calculations. These calculations were performed on relaxed structure models based on the STEM images. Accuracy of the structure models was tested using STEM image simulations. Two-photon time-resolved photo luminescence (2P-TRPL) measurements are carried out providing high-spatial resolution charge-carrier lifetime profiles across the interfaces. Numerical simulation are the carried out to extract recombination velocity at the grain boundaries and correlate to DFT calculations.

Figure 1 shows STEM image of the (111) $5^{\circ}$ bi-crystal interface. The low-angle tilt grain boundary is composed of discrete evenly-spaced $(\sim 5 \mathrm{~nm})$ dislocations. Two-dimensional cross-sectional lifetime maps of the interface are shown in Figure 2, as measured via 2P-TRPL. The PL intensity decay curves were fitted with two exponential functions. Reduction of the lifetimes is evident near the interface, which can be attributed to higher density of recombination sites at the grain boundary. Chlorine chemical treatment as well as Cd-anneal were performed on this grain boundary and the effects on the carrier lifetimes will be presented.

Figure 3 shows atomic-column resolved XEDS map overlaid on HAADF image of (111) $2^{\circ}$ symmetrical $+180^{\circ}$ twist CdTe bicrystal. This grain boundary example shows that in certain orientations a possibility of forming well-ordered uniform structure, instead of localized defects, is possible. Angular-momentum resolved local DOS (LDOS) of the interface is shown in Figure 4. Besides the unusual Te spd-orbital hybridization it can be seen that the structure has high density of midgap states as compared to CdTe bulk 
region which will have important effects on carrier recombination and transport across such boundaries. [4]

\section{References:}

[1] NREL. Best Research-Cell Efficiencies. 2016; Available from:

http://www.nrel.gov/ncpv/images/efficiency_chart.jpg.

[2] Shockley, W. and H.J. Queisser, Detailed Balance Limit of Efficiency of p-n Junction.

[3] Paulauskas, T. et al, Applied Physics Letters, (2014).

[4] We acknowledge funding from the DoE Sunshot program under contract \# DOE DEEE005956.

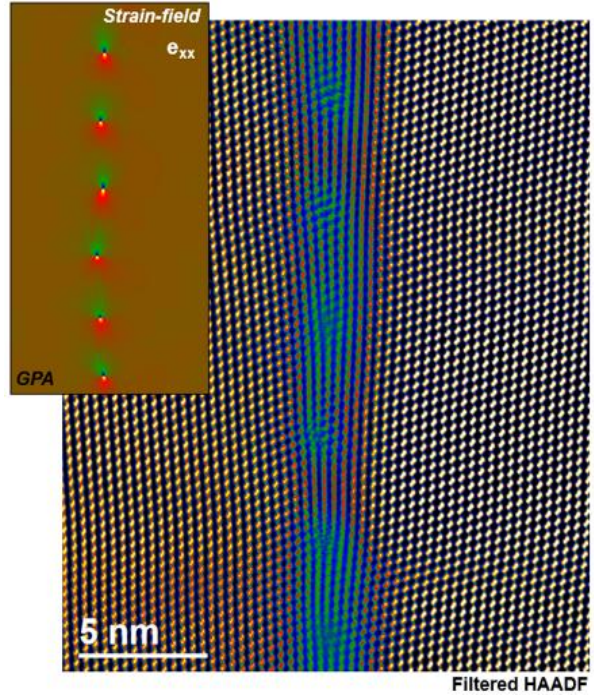

Figure 2: HAADF image of the (111) $5^{\circ}$ bicrystal interface. An array of mixed edgescrew character dislocations are seen along the interface. Inset show geometrical phase analysis (GPA) strain map.

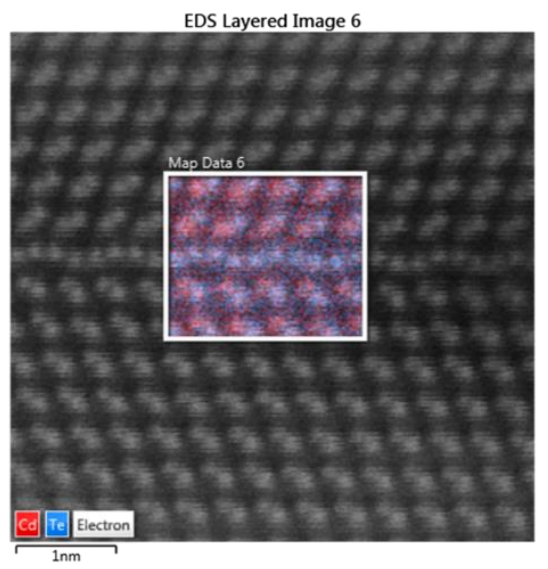

Figure 3: Atomic-column resolved $\mathrm{X}$-ray map of $(111) 2^{\circ}+180^{\circ}$ twist interface. Te monolayer can be seen at the interface. Red Cd-L, blue Te-L X-ray signal.
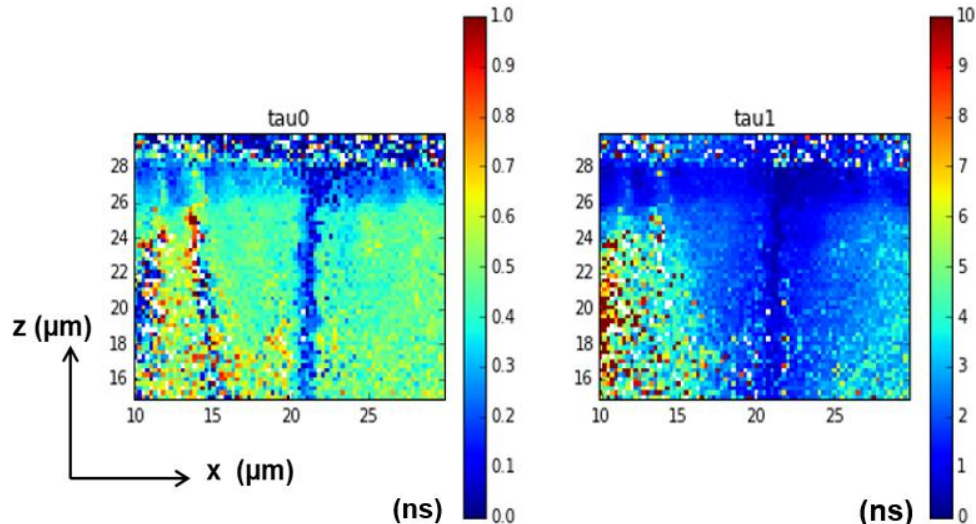

Figure 1: Cross-sectional lifetime map of the bicrystal in Figure 1. Lifetimes (in ns) tau0 and tau 1 present the decay fits using two exponentials. The top surface of crystal is at $\mathrm{z}=28$.

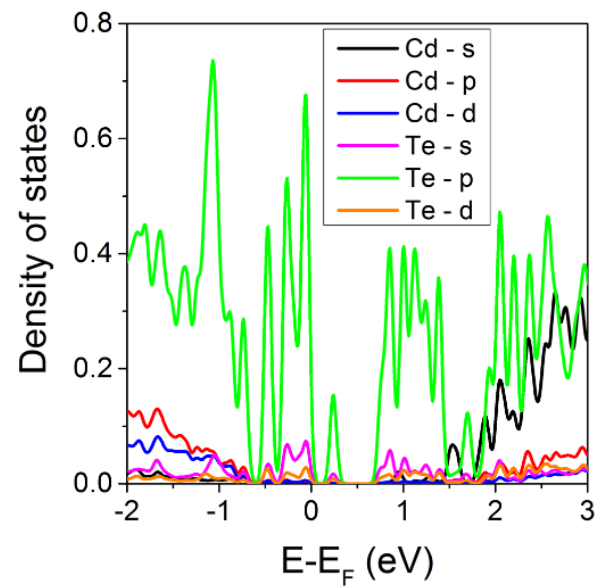

Figure 4: Angular-momentum resolved local DOS of the interface. Te atoms and the first Cd layer above and below the Te monolayer are shown. 